\title{
Gamma-gamma physics and meson transition form factors
}

\author{
Andrzej Kupsc ${ }^{1,2, a}$ \\ ${ }^{1}$ Department of Physics and Astronomy, Uppsala University, Box 516, SE-75120 Uppsala, Sweden \\ ${ }^{2}$ National Centre for Nuclear Research, Pasteura 7, 02-093 Warsaw, Poland
}

\begin{abstract}
I summarize recent experimental results for two photon production of mesons. These processes include the neutral pion, $\eta$ and $\eta^{\prime}$ transition form factors and two photon production of pseudoscalar meson pairs. In addition I discuss the related hadronic and radiative processes. All these processes are attracting attention of experiment and theory due their relevance for the hadronic light-by-light contribution to the muon anomalous magnetic moment.
\end{abstract}

\section{Introduction}

Two photon interactions with mesons and mesonic systems are an important ingredient in the detailed understanding of the nature of mesons and their underlying quark and gluon structure. Presently the main motivation of the related studies is determination of hadronic contribution to the muon anomalous magnetic moment $(g-2)$.

It is now clear that direct searches for new particles at the high-energy accelerators must be complemented by low-energy high-precision measurements. One very promising observable is the muon anomalous magnetic moment [1]. The persistent deviation of 3-4 standard deviations between Standard Model (SM) predictions [2, 3] and the precision BNL experiment [4] has motivated a new measurement at Fermilab with the 0.14 ppm accuracy [5]. With the new experiment under way it is necessary to improve the SM prediction correspondingly. The most difficult to determine precisely is the contribution from hadrons. At present the most reliable way to account for the hadronic contributions to $(g-2)$ is a data-driven approach based on dispersion theory. This first-principle approach connects the hadronic quantum fluctuations that enter $(g-2)$ to other precisely measured observables. The relevant hadronic contributions to $(g-2)$ come from the hadronic vacuum polarization (HVP) and from hadronic light-by-light scattering (HLbL). The HVP contribution is directly related via the dispersion theory to the total cross section of $e^{+} e^{-} \rightarrow$ hadrons [1]. The HLbL contribution requires a much more complicated dispersive framework [68]. The numerically most important contributions to HLbL come from the neutral pion-pole diagram $[9,10]$, poles of other light neutral pseudoscalar mesons $\left(P=\eta, \eta^{\prime}\right)$ and two pion contributions [11]. The necessary input to calculate the pseudoscalar meson pole contribution to $(g-2)$ are transition form factors (TFFs) of the corresponding pseudoscalar mesons: Fig. 1(a). In principle the TFFs could

\footnotetext{
ae-mail: Andrzej.Kupsc@ physics.uu.se
}

be measured experimentally and the status of the recent experiments is presented in the next section. Many of the recent precision experimental studies of the TFFs were initially motivated by the searches for a dark photon [12]. Recent estimates of the $\pi^{0}, \eta$ and $\eta^{\prime}$ poles contribution to HLbL using directly the experimental data on the TFFs are given e.g. in Refs. [10, 13, 14]. An alternative method to determine the TFFs uses large-statistics data sets on hadronic and radiative processes and dispersion relations. For the $\pi^{0}$ TFF such project has been just finished [15, 16] and for $\eta$ and $\eta^{\prime}$ it is ongoing [17]. For the dispersive $\pi^{0}$ TFF determination the most important experimental inputs are pion-pion $p$-wave phase shifts and the cross section for $e^{+} e^{-} \rightarrow \pi^{+} \pi^{-} \pi^{0}$, as discussed in Sec. 4. In the dispersive framework for HLbL [7] the next-important contribution is related to the processes where two-photon couple to a pseudoscalar meson pair shown in Fig. 1(b). Here the processes with pion pair production $P_{1} P_{2}=\pi^{+} \pi^{-}, \pi^{0} \pi^{0}$ dominate. The contributions to the blob can include exchanges of scalar and tensor meson resonances in the $s$-channel and pseudoscalar and vector mesons in the $t$-channel. The two photon processes are discussed in Sec. 3 .

\section{Transition Form Factors}

A transition form factor $\mathcal{F}_{P}\left(q_{1}^{2}, q_{2}^{2}\right)$ is a scalar function of the four-momentum transfer squared of the virtual photons $\left(q_{1,2}^{2}\right)$ describing the vertex in Fig. 1(a) and defined as [18, 19]:

$$
\mathcal{A}\left(P \rightarrow \gamma^{*} \gamma^{(*)}\right)=q_{1}^{\mu} \epsilon_{1}^{v} q_{2}^{\alpha} \epsilon_{2}^{\beta} \epsilon_{\mu \nu \alpha \beta} \mathcal{F}_{P}\left(q_{1}^{2}, q_{2}^{2}\right)
$$

and

$$
\frac{m_{P}^{3}}{64 \pi}\left|\mathcal{F}_{P}(0,0)\right|^{2}=\Gamma(P \rightarrow \gamma \gamma),
$$

where $\Gamma(P \rightarrow \gamma \gamma)$ is the radiative width of the meson $P$. The most precise measurements of the $\pi^{0}$ radiative decay 
width use Primakoff process, photoproduction of pseudoscalar mesons in electric field of nucleus. The value from the PrimEx-I experiment is $(7.82 \pm 0.14 \pm 0.17) \mathrm{eV}$ [20], with a total uncertainty of $2.8 \%$, is the most precise published measurement. A new preliminary result with an improved accuracy from PrimEx-II is $\Gamma\left(\pi^{0} \rightarrow\right.$ $\gamma \gamma)=(7.74 \pm 0.06 \pm 0.12) \mathrm{eV}[21]$. This result should be compared to the Chiral Perturbation Theory prediction of $\Gamma\left(\pi^{0} \rightarrow \gamma \gamma\right)=(8.09 \pm 0.11) \mathrm{eV}[22]$.

For the $\eta$ meson the best result for $\Gamma(\eta \rightarrow \gamma \gamma)$ is determined from the cross section of the $e^{+} e^{-} \rightarrow e^{+} e^{-} P$ process at c.m. energy of $1 \mathrm{GeV}$ by the KLOE- 2 experiment where the final electrons escape the detector at low scattering angles. This cross section is dominated by the contributions from photons with virtualities close to zero, leading to $4.6 \%$ accuracy for the $\eta$ radiative width: $(520 \pm 20 \pm 13)$ $\mathrm{eV}$ [23].

The dependence on the virtualities of the two photons are usually discussed using a normalized transition form factor:

$$
F_{P}\left(q_{1}^{2}, q_{2}^{2}\right)=\frac{\mathcal{F}_{P}\left(q_{1}^{2}, q_{2}^{2}\right)}{\mathcal{F}_{P}(0,0)} .
$$

The photons could be on the mass shell, spacelike or timelike. A quantity often considered in the context of transition form factors is the form factor slope defined as

$$
b_{P}=\left.\frac{\partial F_{P}\left(q^{2}, 0\right)}{\partial q^{2}}\right|_{q^{2}=0} .
$$

For $\pi^{0}$ it is expressed using a dimensionless parameter: $a_{\pi}=b_{\pi^{0}} / m_{\pi^{0}}^{2}$. The best experimental result for $a_{\pi}$ is from spacelike TFF measurement by CLEO: $a_{\pi}=(3.26 \pm$ $0.26 \pm 0.26) \cdot 10^{-2}[24]$. This value can be compared to $a_{\pi}=(3.15 \pm 0.09) \cdot 10^{-2}$ from recent dispersive calculations based only on hadronic data $[15,16]$. The slope definition can be generalized to

$$
b_{P}\left(q_{2}^{2}\right)=\left.\frac{\partial \ln \left|F_{P}\left(q_{1}^{2}, q_{2}^{2}\right)\right|}{\partial q_{1}^{2}}\right|_{q_{1}^{2}=0},
$$

relevant for Dalitz decays of vector mesons like $\phi \rightarrow$ $\pi^{0} e^{+} e^{-}$. Experimental data on the slope parameters for the slopes $b_{\pi^{0}}\left(q^{2}\right)$ (for $q^{2}=m_{\omega}^{2}$ and $q^{2}=m_{\phi}^{2}$ ) and the $b_{\eta}\left(q^{2}\right)$ (for $q^{2}=0$ and $q^{2}=m_{\phi}^{2}$ ) are shown in Table 1 .

An illustration of the $\pi^{0}$ and $\eta$ TFFs is provided in Fig. 2 based on a simplified hadronic phenomenological model [19]. In principle the necessary information could be provided by precision data on the TFFs for arbitrary pairs of photon virtualities. The separate kinematical regions for TFFs shown in Fig. 2 are probed by the following processes:

1. The space like region with $q_{1}^{2}, q_{2}^{2} \leq 0$ (virtualities of both photons are zero or spacelike) is probed by Primakoff effect or $e^{+} e^{-} \rightarrow e^{+} e^{-} P$ where two photons fuse to form a pseudoscalar meson. Analyses to extract single off shell TFF $\left(q_{1}^{2}<0\right.$ and $\left.q_{2}^{2}=0\right)$ for $\pi^{0}$ are underway by BESIII with preliminary results shown in Ref. [34] and by KLOE-2 [35]. Recently the BaBar Collaboration has presented the first measurement of the doubly off-shell $\eta^{\prime}$ TFF [36].

\begin{tabular}{lll}
\hline \multicolumn{3}{c}{$b_{\pi^{0}}\left(q^{2}\right)\left[\mathrm{GeV}^{-2}\right]$} \\
\hline$b_{\pi^{0}}\left(m_{\omega}^{2}\right)$ & $2.4 \pm 0.2$ & Lepton-G [25] \\
$b_{\pi^{0}}\left(m_{\omega}^{2}\right)$ & $2.24 \pm 0.06 \pm 0.02$ & NA60 [26] \\
$b_{\pi^{0}}\left(m_{\omega}^{2}\right)$ & $2.223 \pm 0.026 \pm 0.037$ & NA60 [27] \\
$b_{\pi^{0}}\left(m_{\omega}^{2}\right)$ & $1.99 \pm 0.21$ & A2 [28] \\
\hline \multicolumn{3}{c}{$b_{\eta}\left(q^{2}\right)\left[\mathrm{GeV}^{-2}\right]$} \\
\hline$b_{\eta}(0)$ & $1.9 \pm 0.4$ & Lepton-G [29] \\
$b_{\eta}(0)$ & $1.42 \pm 0.21$ & CELLO [24] \\
$b_{\eta}(0)$ & $1.95 \pm 0.17 \pm 0.05$ & NA60 [26] \\
$b_{\eta}(0)$ & $1.92 \pm 0.35 \pm 0.13$ & CB/TAPS [30] \\
$b_{\eta}(0)$ & $1.934 \pm 0.067 \pm 0.050$ & NA60 [27] \\
$b_{\eta}(0)$ & $1.97 \pm 0.11$ & A2 [28] \\
$b_{\eta}\left(m_{\phi}^{2}\right)$ & $3.8 \pm 1.8$ & SND [31] \\
$b_{\eta}\left(m_{\phi}^{2}\right)$ & $1.17 \pm 0.10 \pm 0.07$ & KLOE-2 [32] \\
$b_{\pi^{0}}\left(m_{\phi}^{2}\right)$ & $2.02 \pm 0.11$ & KLOE-2 [33] \\
\hline
\end{tabular}

Table 1. Summary of the experimental data on the $\pi^{0}$ and $\eta$ TFFs slopes $b_{\pi^{0}}\left(q^{2}\right)$ and $b_{\eta}\left(q^{2}\right)$.

(a)

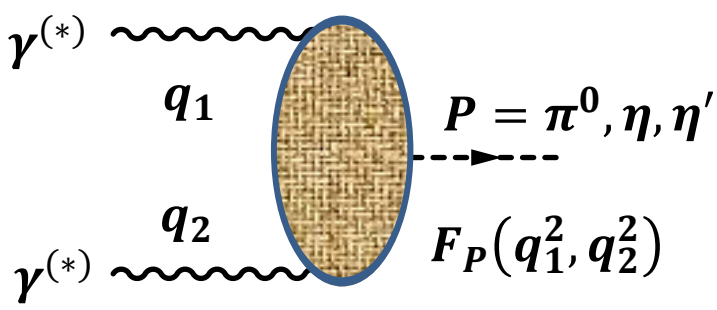

(b)

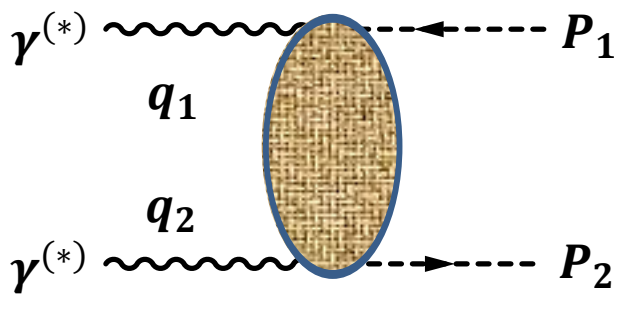

Figure 1. (a): $P \gamma^{*} \gamma^{*}$ vertex - a neutral pseudoscalar meson transition form factor; (b): $P_{1} P_{2} \gamma^{*} \gamma^{*}$ vertex - two photon production of a pseudoscalar meson pair.

2. $P \rightarrow \gamma e^{+} e^{-}$covers $4 m_{P}^{2}>q_{1}^{2}>4 m_{e}^{2}, q_{2}^{2}=0$ region. For $\pi^{0} \rightarrow \gamma e^{+} e^{-}$this process was studied by $\mathrm{NA} 62[37]-a_{\pi}=(3.68 \pm 0.51 \pm 0.25) \cdot 10^{-2}$ and $\mathrm{A} 2[38]-a_{\pi}=(3 \pm 1) \cdot 10^{-2}$. The single Dalitz decay of the $\eta$ meson was recently studied by the A2 Collaboration $\left(\eta \rightarrow e^{+} e^{-} \gamma\right)$ [28] and the NA60 Collaboration [27] $\left(\eta \rightarrow \mu^{+} \mu^{-} \gamma\right)$. The corresponding results for the $b_{\eta}(0)$ slope parameter are given in Table. 1. The first observation of $\eta^{\prime} \rightarrow \gamma e^{+} e^{-}$was reported by BESIII [39].

3. $P \rightarrow 2 e^{+} 2 e^{-}$happens in the hardly visible tiny region between the positive axes and the hyperbola. 


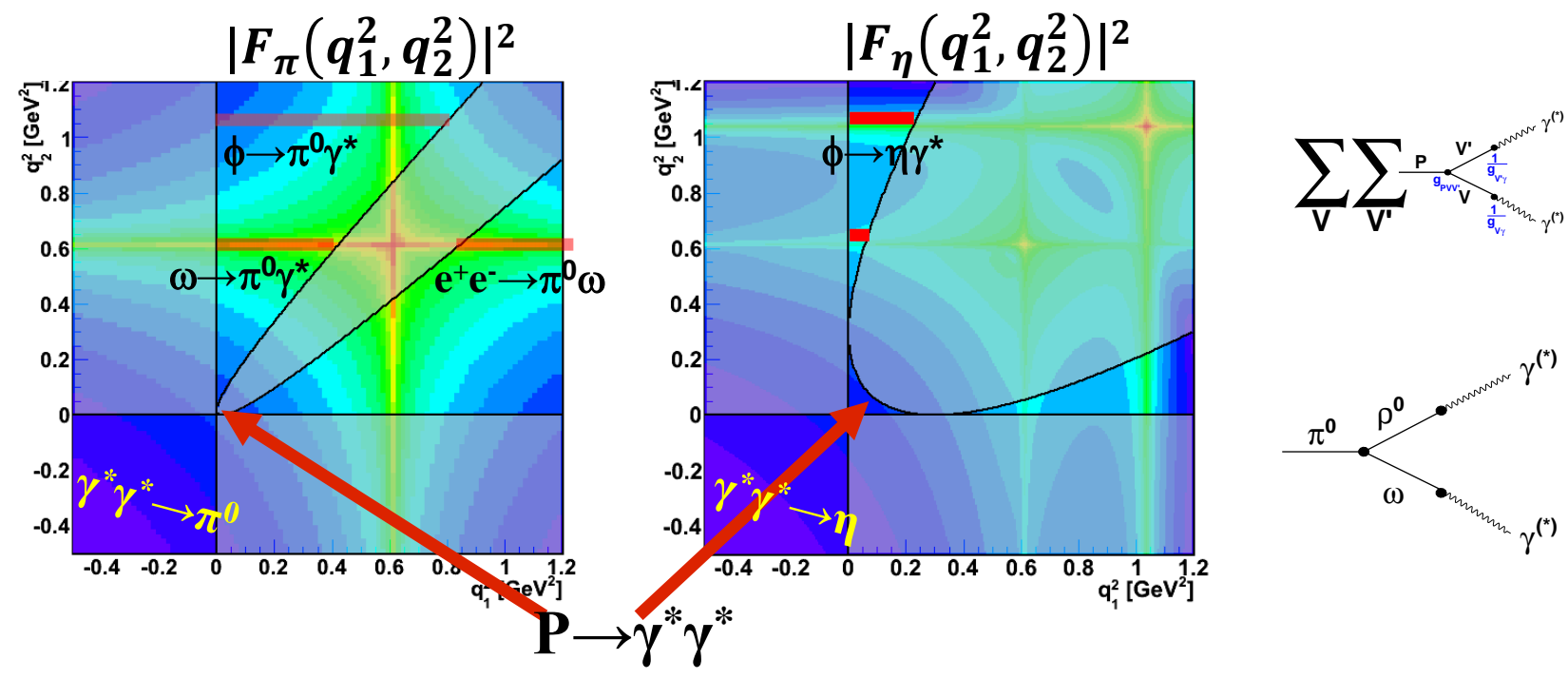

Figure 2. Kinematical regions of the $\pi^{0}$ and $\eta$ TFFs as function of the photon virtualities $q_{1}^{2}, q_{2}^{2}$. The ridges are caused by the direct coupling of photons to vector mesons according to the simplest version of the Vector Meson Dominance model (diagrams to the left). The greyish regions top left, bottom right and inside of the hyperbola are not experimentally accessible.

The process corresponds to the case when the real photons are replaced by two dileptons and one explores the doubly virtual case with both virtualities timelike. In Ref. [40] KLOE-2 has reported first observation of $\eta \rightarrow 2 e^{+} 2 e^{-}$.

4. $e^{+} e^{-} \rightarrow P \gamma$ covers $q_{1}^{2}>4 m_{P}^{2}, q_{2}^{2}=0$ region. In principle this process is a part of the hadronic cross section contributing to HVP. In particular $e^{+} e^{-} \rightarrow \pi^{0} \gamma$ represents HVP process with the lowest threshold at the c.m. energy of $m_{\pi^{0}}$. However, the cross section is low and therefore the process is mainly of importance for the HLbL contribution. So far it was studied only in the scan experiments where the collider c.m. energy is tuned to a specific $q_{1}$ value to measure the cross section. In the most relevant/important region for the muon $(g-2)$ this is only possible at the Novosibirsk VEPP-2000 collider. The most recent results are from SND detector in the $0.60-1.38 \mathrm{GeV}$ [41] and $1.075-2 \mathrm{GeV}$ [42] ranges.

5. $e^{+} e^{-} \rightarrow \gamma^{*} \rightarrow P e^{+} e^{-}$corresponds to the colourful regions to the left and right of the hyperbolas. It was studied directly when the c.m. of the reaction was fixed to a narrow vector meson mass such as $\omega, \phi$ or $J / \psi$ resonances. Detailed analysis of the electron-positron invariant mass distribution in the $\phi \rightarrow P e^{+} e^{-}$decay were performed at KLOE$2[32,33]$ and $J / \psi \rightarrow P e^{+} e^{-}$at BESIII [43]. The process can be studied in vector meson decays regardless the production method: in photoproduction by the A2 Collaboration: $\omega \rightarrow \pi^{0} e^{+} e^{-}$[28] and in hadronic processes by the NA60 Collaboration: $\omega \rightarrow \mu^{+} \mu^{-} \pi^{0}$ [27]. The $\pi^{0}$ TFF was extracted for $q_{1}^{2}=m_{\omega}^{2}$ and $4 m_{l}^{2}<q_{2}^{2}<\left(m_{\omega}-m_{\pi^{0}}\right)^{2}$ including the $b_{\pi^{0}}\left(m_{\omega}^{2}\right)$ values given in Table 1 . The directly related process $e^{+} e^{-} \rightarrow \omega \pi^{0}$ allows to probe the $\left(m_{\omega}+m_{\pi^{0}}\right)^{2}<q_{2}^{2}$ region with the most recent results from the SND Collaboration [44].

In addition the decays of the pseudoscalar mesons into lepton-antilepton pair, $P \rightarrow l^{+} l^{-}$, involve two photon intermediate state. The branching fraction values provide a sensitive test for the TFFs with doubly virtual photons since the process involves a loop where $q_{1}^{2}$ and $q_{2}^{2}$ can take arbitrary values. The decays into the electronpositron pair are additionally suppressed by the (approximate) electron helicity conservation. The puzzle of the observed enhanced $\pi^{0} \rightarrow e^{+} e^{-}$decay rate $[45,46]$ is most likely explained by the higher order radiative corrections [47]. The decays $\eta^{(\prime)} \rightarrow e^{+} e^{-}$were not yet observed. The best upper limits come from formation experiments $e^{+} e^{-} \rightarrow \gamma^{*} \gamma^{*} \rightarrow P$ at VEPP-2000. The $90 \%$ C.L. upper limits are $B\left(\eta \rightarrow e^{+} e^{-}\right)<7 \cdot 10^{-7}$ [48] and $B\left(\eta^{\prime} \rightarrow e^{+} e^{-}\right)<5.6 \cdot 10^{-9}[49]$.

\section{Two photon production of pseudoscalar meson pairs}

Structure of two photon production of meson pairs $\gamma^{(*)} \gamma^{(*)} \rightarrow P_{1} P_{2}$, where $P_{1} P_{2}=$ $\pi^{+} \pi^{-}, \pi^{0} \pi^{0}, \pi^{+} \eta, \eta \eta, K^{+} K^{-}, K_{S} K_{S}, \ldots$, is much more complicated than TFFs and can consist up to five independent tensors [50]. Experimental studies of such processes can be performed in reaction $e^{+} e^{ \pm} \rightarrow e^{+} e^{ \pm} \gamma^{*} \gamma^{*} \rightarrow e^{+} e^{ \pm} P_{1} P_{2}$ or in Primakoff effect $\gamma N \rightarrow P_{1} P_{2} N$. The cross section for two photon processes at an electron positron collider is given in equivalent photon approximation as [51]:

$$
\sigma\left(e^{+} e^{-} \rightarrow e^{+} e^{-} P_{1} P_{2}\right)=\frac{1}{L_{e e}} \int \frac{d L}{d W} \sigma_{\gamma \gamma \rightarrow P_{1} P_{2}}(W) d W,
$$




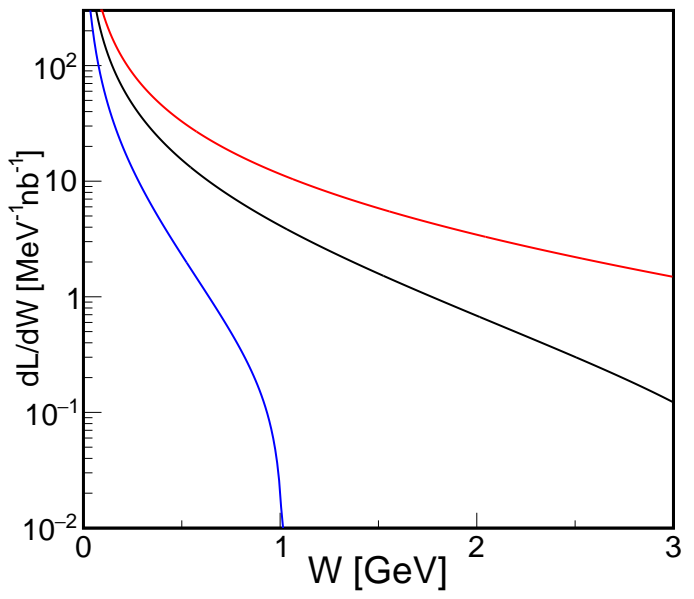

Figure 3. Differential $\gamma \gamma$ luminosity $d L / d W$ in $e^{+} e^{-}$for $L_{e e}=1$ $\mathrm{fb}^{-1}$ as function of c.m. energy of the two photons $W$ : red line $\sqrt{s}=10 \mathrm{GeV}$ (Belle), black line $-\sqrt{s}=3.77 \mathrm{GeV}$ (BESIII) and blue line $\sqrt{s}=1.02 \mathrm{GeV}$ (KLOE-2).

where $W$ is the invariant mass of the $P_{1} P_{2}$ system, $d L / d W$ is differential luminosity of the $\gamma \gamma$ and $L_{e e}$ is the integrated luminosity for the $e^{+} e^{-}$collisions. The differential luminosity for $\gamma \gamma \rightarrow P_{1} P_{2}$ increases with c.m. energy of the $e^{+} e^{-}$collisions $\sqrt{s}$ as illustrated in Fig. 3. The differential luminosities are drawn for $\sqrt{s}=1.02,3.77$ and $10 \mathrm{GeV}$.

The experimental studies of the two photon processes had been focused so far on the easiest case with quasireal photons. It corresponds to the situation where none of the outgoing electrons is measured. The recent, most accurate and comprehensive results are from the Belle experiment: $\pi^{0} \pi^{0}$ [52, 53] $\pi^{+} \pi^{-}$[54, 55], $\pi^{0} \eta$ [56], $\eta \eta$ [57], $K^{+} K^{-}[54,58]$, and $K_{s}^{0} K_{s}^{0}$ [59]. The data allows one to determine radiative widths of $C=+1$ scalar and tensor resonances. The first measurements of $\pi^{0}$ and $K_{s}^{0}$ pairs from a singly off-shell photon process $\gamma \gamma^{(*)} \rightarrow P \bar{P}[60,61]$ were reported by the Belle Collaboration, while the production of the $\pi^{+} \pi^{-}$pairs is currently analyzed at BESIII [62].

\section{Other related data}

Both for the spacelike and timelike region it is unlikely to obtain doubly virtual data with appropriate precision in the near future. The processes are suppressed by powers of the fine-structure constant and by photon propagators that scale inversely with the photon virtualities. An alternative to the direct experimental determination of TFFs is given by dispersion theory $[9,15,16]$. The $\pi^{0}$ TFF is related to pion vector form factor and to three-pion-photon amplitude. Such contribution could be represented by isovector and isoscalar photons as illustrated in a simplified Vector Meson Dominance (VMD) diagrams in Fig. 2(left). The three-pion-photon amplitude for arbitrary photon virtualities and arbitrary values of the two-pion Mandelstam variables are needed. The dependence on the photon virtuality must be determined from data whereas the dependence on the Mandelstam variables is dominated by pion rescattering and can be predicted by dispersion theory based on the available very precise pion-pion phase shifts [63]. The experimental input for this approach to the pion TFF is given by the studies of the $e^{+} e^{-} \rightarrow \pi^{+} \pi^{-} \pi^{0}$ reaction cross section and the dynamical variables in this three body process. In addition to the cross section studies in the context of HVP, the analysis of $\omega, \phi \rightarrow \pi^{+} \pi^{-} \pi^{0}$ decay dynamics provides a valuable cross check of the dispersive formalism. Until recently, surprisingly little information had been available on the $\omega \rightarrow \pi^{+} \pi^{-} \pi^{0}$ Dalitz plot. First observation of a deviation from $P$-wave phase space consistent with $\rho$ meson contribution was reported by WASAat-COSY [64]. Recently high statistics result from BESIII was released [65], with accuracy allowing to test dispersive calculations $[66,67]$.

For $\eta$ and $\eta^{\prime}$ the relation between radiative and Dalitz decays is obtained in a simple, model independent way. The precision experimental data on the radiative decays $\eta^{(\prime)} \rightarrow \gamma \pi^{+} \pi^{-}$are used to predict the form factors in in the region relevant to $\eta^{(\prime)} \rightarrow \gamma e^{+} e^{-}$[68-70]. The experimental input for $\eta \rightarrow \gamma \pi^{+} \pi^{-}$is provided by WASA-atCOSY [71] and KLOE-2 [72] and for $\eta^{\prime} \rightarrow \gamma \pi^{+} \pi^{-}$by BESIII [73]. To extend the predictions to other kinematic regions of the $\eta$ and $\eta^{\prime}$ TFFs, the data for other processes are needed e.g. $e^{+} e^{-} \rightarrow \eta^{(\prime)} \pi^{+} \pi^{-}$(recent measurements from SND [74] and BaBar [75]) or decays $\eta^{(\prime)} \rightarrow \pi^{+} \pi^{-} e^{+} e^{-}$(recent study of the $\eta$ decay at WASA-at-COSY [76]).

\section{References}

[1] F. Jegerlehner, A. Nyffeler, Phys. Rept. 477, 1 (2009)

[2] M. Davier, A. Hoecker, B. Malaescu, Z. Zhang, Eur. Phys. J. C71, 1515 (2011), [Erratum: Eur. Phys. J.C72,1874(2012)]

[3] K. Hagiwara, R. Liao, A.D. Martin, D. Nomura, T. Teubner, J. Phys. G38, 085003 (2011)

[4] G.W. Bennett et al. (Muon g-2), Phys. Rev. D73, 072003 (2006)

[5] A. Chapelain (Muon g-2), EPJ Web Conf. 137, 08001 (2017)

[6] G. Colangelo, M. Hoferichter, M. Procura, P. Stoffer, JHEP 09, 091 (2014)

[7] G. Colangelo, M. Hoferichter, B. Kubis, M. Procura, P. Stoffer, Phys. Lett. B738, 6 (2014)

[8] V. Pauk, M. Vanderhaeghen, Phys. Rev. D90, 113012 (2014)

[9] M. Hoferichter, B. Kubis, S. Leupold, F. Niecknig, S.P. Schneider, Eur. Phys. J. C74, 3180 (2014)

[10] A. Nyffeler, Phys. Rev. D94, 053006 (2016)

[11] G. Colangelo, M. Hoferichter, M. Procura, P. Stoffer, JHEP 04, 161 (2017)

[12] M. Pospelov, Phys. Rev. D80, 095002 (2009)

[13] P. Masjuan, P. Sanchez-Puertas, Phys. Rev. D95, 054026 (2017)

[14] H. Czyż, P. Kisza, S. Tracz, Phys. Rev. D97, 016006 (2018) 
[15] M. Hoferichter, B.L. Hoid, B. Kubis, S. Leupold, S.P. Schneider, Phys. Rev. Lett. 121, 112002 (2018)

[16] M. Hoferichter, B.L. Hoid, B. Kubis, S. Leupold, S.P. Schneider, JHEP 10, 141 (2018)

[17] C.W. Xiao, T. Dato, C. Hanhart, B. Kubis, U.G. Meißner, A. Wirzba (2015)

[18] L.G. Landsberg, Phys. Rept. 128, 301 (1985)

[19] E. Czerwinski, S. Eidelman, C. Hanhart, B. Kubis, A. Kupsc, S. Leupold, P. Moskal, S. Schadmand, MesonNet Workshop on Meson Transition Form Factors (2012), arXiv:hep-ph/1207.6556

[20] I. Larin et al. (PrimEx), Phys. Rev. Lett. 106, 162303 (2011)

[21] A.H. Gasparian, PoS CD15, 048 (2016)

[22] K. Kampf, B. Moussallam, Phys. Rev. D79, 076005 (2009)

[23] D. Babusci et al. (KLOE-2), JHEP 01, 119 (2013)

[24] H.J. Behrend et al. (CELLO), Z. Phys. C49, 401 (1991)

[25] R.I. Dzhelyadin et al., Phys. Lett. 102B, 296 (1981), [JETP Lett.33,228(1981)]

[26] R. Arnaldi et al. (NA60), Phys. Lett. B677, 260 (2009)

[27] R. Arnaldi et al. (NA60), Phys. Lett. B757, 437 (2016)

[28] P. Adlarson et al., Phys. Rev. C95, 035208 (2017)

[29] R.I. Dzhelyadin et al., Phys. Lett. 94B, 548 (1980), [Yad. Fiz.32,998(1980)]

[30] H. Berghauser et al., Phys. Lett. B701, 562 (2011)

[31] M.N. Achasov et al., Phys. Lett. B504, 275 (2001)

[32] D. Babusci et al. (KLOE-2), Phys. Lett. B742, 1 (2015)

[33] A. Anastasi et al. (KLOE-2), Phys. Lett. B757, 362 (2016)

[34] C.F. Redmer (BESIII), Measurement of meson transition form factors at BESIII, in 13th Conference on the Intersections of Particle and Nuclear Physics (CIPANP 2018) Palm Springs, California, USA, May 29-June 3, 2018 (2018)

[35] S. Giovannella (KLOE-2), EPJ Web Conf. 166, 00011 (2018)

[36] J.P. Lees et al. (BaBar), Phys. Rev. D98, 112002 (2018)

[37] C. Lazzeroni et al. (NA62), Phys. Lett. B768, 38 (2017)

[38] P. Adlarson et al. (A2), Phys. Rev. C95, 025202 (2017)

[39] M. Ablikim et al. (BESIII), Phys. Rev. D92, 012001 (2015)

[40] F. Ambrosino et al. (KLOE, KLOE-2), Phys. Lett. B702, 324 (2011)

[41] M.N. Achasov et al. (SND), Phys. Rev. D93, 092001 (2016)

[42] M.N. Achasov et al., Phys. Rev. D98, 112001 (2018)

[43] M. Ablikim et al. (BESIII), Phys. Rev. D89, 092008 (2014)

[44] M.N. Achasov et al., Phys. Rev. D94, 112001 (2016)
[45] E. Abouzaid et al. (KTeV), Phys. Rev. D75, 012004 (2007)

[46] A.E. Dorokhov, M.A. Ivanov, Phys. Rev. D75, 114007 (2007)

[47] T. Husek, K. Kampf, J. Novotný, Eur. Phys. J. C74, 3010 (2014)

[48] M.N. Achasov et al. (SND), Phys. Rev. D98, 052007 (2018)

[49] M.N. Achasov et al., Phys. Rev. D91, 092010 (2015)

[50] W.A. Bardeen, W.K. Tung, Phys. Rev. 173, 1423 (1968), [Erratum: Phys. Rev.D4,3229(1971)]

[51] S.J. Brodsky, T. Kinoshita, H. Terazawa, Phys. Rev. D4, 1532 (1971)

[52] S. Uehara et al. (Belle), Phys. Rev. D78, 052004 (2008)

[53] S. Uehara et al. (Belle), Phys. Rev. D79, 052009 (2009)

[54] H. Nakazawa et al. (Belle), Phys. Lett. B615, 39 (2005)

[55] T. Mori et al. (Belle), J. Phys. Soc. Jap. 76, 074102 (2007)

[56] S. Uehara et al. (Belle), Phys. Rev. D80, 032001 (2009)

[57] S. Uehara et al. (Belle), Phys. Rev. D82, 114031 (2010)

[58] K. Abe et al. (Belle), Eur. Phys. J. C32, 323 (2003)

[59] S. Uehara et al. (Belle), PTEP 2013, 123C01 (2013)

[60] M. Masuda et al. (Belle), Phys. Rev. D93, 032003 (2016)

[61] M. Masuda et al. (Belle), Phys. Rev. D97, 052003 (2018)

[62] Y. Guo (BESIII), Nucl. Part. Phys. Proc. 294-296, 153 (2018)

[63] R. Garcia-Martin, R. Kaminski, J.R. Pelaez, J. Ruiz de Elvira, F.J. Yndurain, Phys. Rev. D83, 074004 (2011)

[64] P. Adlarson et al. (WASA-at-COSY), Phys. Lett. B770, 418 (2017)

[65] M. Ablikim et al. (BESIII), Phys. Rev. D98, 112007 (2018)

[66] F. Niecknig, B. Kubis, S.P. Schneider, Eur. Phys. J. C72, 2014 (2012)

[67] I.V. Danilkin, C. Fernández-Ramírez, P. Guo, V. Mathieu, D. Schott, M. Shi, A.P. Szczepaniak, Phys. Rev. D91, 094029 (2015)

[68] F. Stollenwerk, C. Hanhart, A. Kupsc, U.G. Meissner, A. Wirzba, Phys. Lett. B707, 184 (2012)

[69] C. Hanhart, A. Kupsc, U.G. Meissner, F. Stollenwerk, A. Wirzba, Eur. Phys. J. C73, 2668 (2013), [Erratum: Eur. Phys. J.C75 (2015)242].

[70] C. Hanhart, S. Holz, B. Kubis, A. Kupsc, A. Wirzba, C.W. Xiao, Eur. Phys. J. C77, 98 (2017), [Erratum: Eur. Phys. J.C78(2018)450]

[71] P. Adlarson et al. (WASA-at-COSY), Phys. Lett. B707, 243 (2012)

[72] D. Babusci et al. (KLOE), Phys. Lett. B718, 910 (2013) 
[73] M. Ablikim et al. (BESIII), Phys. Rev. Lett. 120, 242003 (2018)

[74] M.N. Achasov et al., Phys. Rev. D97, 012008 (2018)
[75] J.P. Lees et al. (BaBar), Phys. Rev. D97, 052007 (2018)

[76] P. Adlarson et al., Phys. Rev. C94, 065206 (2016) 